\title{
Abdominal fat pad biopsies exhibit good diagnostic accuracy in patients with suspected transthyretin amyloidosis
}

Hedvig Paulsson Rokke1, Nima Sadat Gousheh¹, Per Westermark², Ole B. Suhr¹, Intissar Anan 1,3, Elisabet Ihse², Björn Pilebro ${ }^{1}$ and Jonas Wixner ${ }^{1 *}$ (D)

\begin{abstract}
Background: The diagnostic accuracy of histopathological detection of transthyretin amyloid (ATTR) by Congo red staining of abdominal fat samples has been questioned since low sensitivity has been reported, especially for patients with ATTR cardiomyopathy. However, the outcome of surgically obtained fat pad biopsies has not yet been evaluated. The aim was to evaluate the diagnostic accuracy of skin punch biopsies from abdominal fat in patients with suspected ATTR amyloidosis.
\end{abstract}

Material and methods: Data were evaluated from patients who had undergone abdominal fat pad biopsies using a skin punch due to suspected amyloidosis from 2006 to 2015. The biopsies had been analysed using Congo red staining to determine the presence of amyloid, and immunohistochemistry or Western blot to determine the type of amyloidosis. The final diagnosis was based on the clinical picture, biopsy results and DNA sequencing. Minimum follow-up after the initial biopsy was 3 years.

Results: Two hundred seventy-four patients (61\% males) were identified, and in 132 (48\%), a final diagnosis of amyloidosis had been settled. The majority (93\%) had been diagnosed with hereditary transthyretin (ATTRv) amyloidosis, and therefore subsequent analyses were focused on these patients. Overall, our data showed a test specificity of 99\% and a sensitivity of $91 \%$. Ninety-eight (94\%) of the patients had neuropathic symptoms at diagnosis, whereas 57 (55\%) had signs of amyloid cardiomyopathy. Subgroup analyses showed that patients with merely neuropathic symptoms displayed the highest test sensitivity of 91\%, whereas patients with pure cardiomyopathy displayed the lowest sensitivity of $83 \%$. However, no significant differences in sensitivity were found between patients with or without cardiomyopathy or between the sexes.

Conclusions: Abdominal fat pad biopsies exhibit good diagnostic accuracy in patients with suspect ATTRv amyloidosis, including patients presenting with cardiomyopathy. In addition, the method enables typing not only of the precursor protein but also of the amyloid fibril type, which is related to the phenotype and to the outcome of the disease.

Keywords: Adipose tissue, Amyloid polyneuropathy, Amyloidosis, hereditary, Biopsy, Cardiomyopathy, restrictive, Techniques and procedures, diagnostic, Transthyretin

*Correspondence: jonas.wixner@umu.se

${ }^{1}$ Department of Public Health and Clinical Medicine, Umeå University, 90187 Umeå, Sweden

Full list of author information is available at the end of the article

\section{Background}

Amyloidosis is an acquired or inherited condition caused by extracellular deposition of insoluble fibrillar protein (amyloid) that is formed by misfolded proteins original author(s) and the source, provide a link to the Creative Commons licence, and indicate if changes were made. The images or other third party material in this article are included in the article's Creative Commons licence, unless indicated otherwise in a credit line to the material. If material is not included in the article's Creative Commons licence and your intended use is not permitted by statutory regulation or exceeds the permitted use, you will need to obtain permission directly from the copyright holder. To view a copy of this licence, visit http://creativecommons.org/licenses/by/4.0/. The Creative Commons Public Domain Dedication waiver (http://creativeco mmons.org/publicdomain/zero/1.0/) applies to the data made available in this article, unless otherwise stated in a credit line to the data. 
that assemble into beta-pleated fibrils. Amyloid formation and deposition can be localized or systemic, and can negatively affect the function of the targeted tissues [1]. Demonstration of amyloid deposits in tissue samples using Congo red staining and polarized light microscopy is the gold standard for diagnosing amyloidosis [2]. Tissue samples can be obtained either from the primarily affected organ (e.g. heart or peripheral nerves), or in the case of systemic amyloidosis, from more easily accessible tissues (e.g. abdominal fat, labial salivary glands or the digestive tract) [3]. The tissue of choice varies between clinics depending on local traditions and access to diagnostic procedures. Typing of the amyloid precursor protein is vital since it has implications on disease pathogenesis and on the choice of therapy [3-5]; this is usually performed by immunohistochemistry, immune electron microscopy, mass spectrometry or Western blot analysis.

Transthyretin (ATTR) amyloidosis is one of the major systemic amyloidoses and is induced by the deposition of misfolded transthyretin (TTR). Hereditary transthyretin (ATTRv) amyloidosis is caused by TTR mutations that decrease the stability of the TTR tetramer, whereas acquired (wild-type) transthyretin (ATTRwt) amyloidosis is related to tetramer destabilization with increasing age and possibly also to a decreased protein aggregate clearance in the elderly [6, 7]. ATTRwt amyloidosis is being increasingly recognized and may affect up to $25 \%$ of individuals over the age of 80 years according to postmortem studies $[8,9]$. Although rare, ATTRv amyloidosis is the most widespread inherited systemic amyloidosis that can be found all over the world with certain clustering areas in, for example, Portugal, Brazil, Mallorca, Sweden and Japan [10, 11]. To date, over 120 amyloidogenic TTR mutations have been demonstrated of which the TTR V30M ( $p . V 50 M)$ is the most common variant $[11,12]$. The disease phenotype is related to the genotype; however, phenotypic variations have also been observed within genotypes. Most genotypes are characterized by neuropathic complications with or without cardiac involvement, although some genotypes are associated with mainly oculoleptomeningeal or cardiac complications [10]. In ATTR amyloidosis, the amyloid fibril type has also been shown to be associated with the disease phenotype. Amyloid deposits containing either a mixture of TTR fragments and full-length TTR (Type A) or full-length TTR only (Type B) have been noted. In ATTRV30M amyloidosis, type A fibrils are generally associated with older age at onset and amyloid cardiomyopathy, whereas type B fibrils are associated with younger age at onset and mainly autonomic and peripheral neuropathic complications [13]. Moreover, the presence of type A fibrils has been linked to a poorer outcome after liver transplantation, which until quite recently has been the only available treatment for ATTRv amyloidosis [14].

In Sweden, we have a long tradition of performing abdominal fat pad biopsies for diagnosing systemic amyloidosis in general and for ATTR amyloidosis in particular [15]. Further, surgically obtained abdominal fat pad biopsies have been the standard diagnostic method at the Amyloidosis Centre in Umeå since 2006. Our clinical experience is that this is a safe and reliable method. However, the diagnostic accuracy of abdominal fat pad biopsies has been questioned in patients with ATTR cardiomyopathy, especially in regard to fine needle aspirates $[16,17]$. Recently, it was suggested that bone scintigraphy (e.g. ${ }^{99 \mathrm{~m}} \mathrm{Tc}-\mathrm{DPD}$ scintigraphy) is a reliable and safe noninvasive method for diagnosing ATTR cardiomyopathy [18]. Therefore, the aim of the current study was to evaluate the diagnostic accuracy of abdominal fat pad biopsies in patients with suspected ATTR amyloidosis, and to explore possible differences in diagnostic specificity and sensitivity across phenotypes.

\section{Methods}

Data were evaluated from all patients who had undergone at least one abdominal fat pad biopsy at the Amyloidosis Centre, Umeå University Hospital between January 2006 and December 2015. This time span was chosen since electronic medical records were introduced at our centre in 2006 and since a follow-up time of at least three years was considered to be sufficient to capture any possibly false negative results. The code TQX10 (punch biopsy) from the Swedish Classification of Health Interventions (KVÅ) version 2019 was used to identify the patients in our medical records in January 2019.

All biopsies had been performed in local anaesthesia using an $8 \mathrm{~mm}$ skin punch (Fig. 1) by physicians (mainly OBS, JW and IA-co-authors) at our outpatient department as per routine clinical practice. After the diagnostic procedure, the biopsies had been stored in a saline solution and later analysed at the Department of Clinical Pathology, Uppsala University Hospital by a dedicated pathologist (PW-co-author). For amyloid diagnosis, parts of the adipose tissue (about $100 \mathrm{~mm}^{3}$ ) were put on a glass slide, cut into small pieces with a pair of scissors and squeeze preparation was obtained with the aid of another slide. The two glass slides were then dried, defatted in acetone, Congo red stained and mounted. The slides were scrutinized in a polarization microscope to determine the presence of even minute deposits of amyloid [19]. Thus, formalin-fixation, paraffin embedding and sectioning were avoided, which allowed for thicker tissue specimens to be obtained. The amyloid type was determined by immunohistochemistry or Western blot analysis as previously described $[15,20]$. 

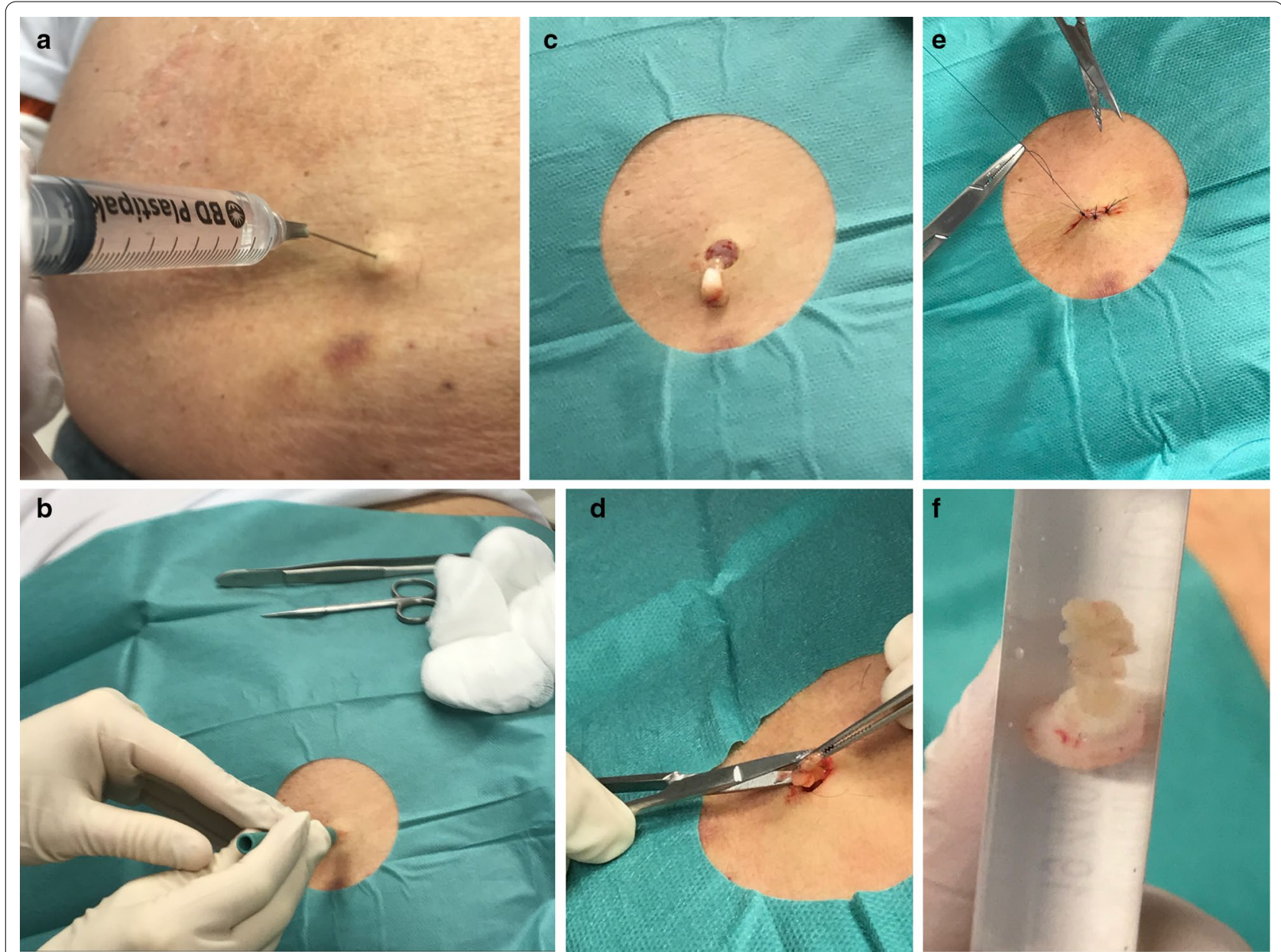

Fig. 1 Abdominal fat pad biopsy procedure. How we perform an abdominal fat pad biopsy using an $8 \mathrm{~mm}$ skin punch. a Local anaesthesia with premixed lidocaine $(10 \mathrm{mg} / \mathrm{ml})$ with adrenaline $(5 \mathrm{\mu g} / \mathrm{ml})$ after disinfection with chlorhexidine $(5 \mathrm{mg} / \mathrm{ml})$. b Skin punch after applying a sterile drape and using sterile gloves. c Biopsy sample. $\mathbf{d}$ Cutting out additional fat using a pair of forceps and a pair of scissors. e Suture with thread size 4- 0 , two simple interrupted sutures and one central vertical mattress suture is usually adequate. $\mathbf{f}$ Final biopsy sample, including skin punch and additional fat, in saline (sodium chloride $9 \mathrm{mg} / \mathrm{ml}$ )

Patients' medical records were reviewed and data on sex, age at disease onset, age and symptoms at the time of the biopsy, TTR genotype (if applicable), outcome of abdominal fat pad or any other tissue biopsies, and amyloid fibril type (if determined) were collected, as well as data from other diagnostic procedures if performed within one year from the biopsy date. Signs of peripheral polyneuropathy had been evaluated with clinical examinations at the Amyloidosis Centre, as well as with nerve conduction studies and electromyograms at the Department of Neurophysiology, Umeå University Hospital. Amyloid cardiomyopathy was considered present if ${ }^{99} \mathrm{~m}$ Tc-DPD scintigraphy was positive (Perugini grade 1-3) [21] and/or if the interventricular septum thickness was $\geq 12 \mathrm{~mm}$ on echocardiogram and no other cause of ventricular hypertrophy was evident. The scintigraphies and the echocardiograms had been performed as per local clinical practice at the Department of Radiology and at the Department of Clinical Physiology, Umeå University Hospital, respectively. ${ }^{99 \mathrm{~m}} \mathrm{Tc}-\mathrm{DPD}$ scintigraphy was introduced at our centre in 2012. Gastrointestinal (GI) symptoms had been inquired for at the Amyloidosis Centre according to our routine clinical practice [22]. Signs of ocular involvement had been evaluated at the Department of Ophthalmology, Umeå University due to visual impairment or as part of our standard clinical followup. The patients were divided into subgroups according to their clinical symptoms/findings (polyneuropathy, cardiomyopathy, GI symptoms and eye complications, respectively).

A final diagnosis of amyloidosis had been established by the treating physician based on the clinical picture, tissue 
biopsy results (sometimes multiple), DNA sequencing, and in some cases, ${ }^{99 \mathrm{~m}} \mathrm{Tc}$-DPD scintigraphy as per routine clinical practice. Demonstration of amyloid deposits in a tissue biopsy was considered the gold standard for diagnosis. Patients with a previously established amyloidosis diagnosis (before 2006) were excluded from further analysis, and individuals with a TTR mutation without any signs of ATTR amyloidosis were considered asymptomatic mutation carriers. Late onset of ATTR amyloidosis was defined as onset of symptoms after the age of 50 years as per clinical practice. TTR mutations are denominated using the single letter amino acid code and the sequence numbering of the mature protein.

Statistical analyses were performed with SPSS versions 25 and 26. Data are expressed as medians and full range. Specificity and sensitivity analyses were based on the outcome of the first fat pad biopsy and the established diagnosis within three years from the initial biopsy. Nominal data were analysed with chi $^{2}$-tests. $p$ values $<0.05$ were regarded as statistically significant.

\section{Results}

\section{Patients and diagnoses}

Two hundred and seventy-six patients had undergone one or more abdominal fat pad biopsy between 2006 and 2015. Two patients were excluded due to lack of clinical data in their medical records, which resulted in 274 patients (61\% males) included in the analyses (see Additional file 1). Baseline patient characteristics, at the time of the first tissue biopsy, are outlined in Table 1. All subjects had some symptom or finding suggestive of amyloidosis. No data on the time between symptom onset and the time of tissue biopsy were available.

Median follow-up after first biopsy was 8 (3-12) years. During the study period, $48(17 \%)$ of the patients had undergone a second abdominal fat pad biopsy and 33 (12\%) had been subjected to a tissue biopsy from another site (e.g. GI tract, heart, skin and kidney). Ultimately, 132 patients had got a final diagnosis of amyloidosis, mainly ATTRv amyloidosis, and 53 had been considered as asymptomatic carriers of a TTR mutation (Table 2). Median age at diagnosis was 63 (22-88) years. Nineteen patients (who had undergone non-diagnostic biopsies for research purposes) were excluded from further analyses due to an established amyloidosis diagnosis prior to 2006 (see Additional file 1).

Typing of the precursor protein (TTR) and the amyloid fibril type was available for 82 (89\%) of the patients with ATTRv amyloidosis and proven amyloid deposits in their first fat pat biopsy. The majority (57\%) of the patients displayed type A fibrils, and no significant difference in fibril type distribution was seen between the sexes $(p=0.36)$.
Table 1 Baseline patient characteristics

\begin{tabular}{|c|c|c|c|}
\hline & Male $(n=167)$ & $\begin{array}{l}\text { Female } \\
(n=107)\end{array}$ & All $(n=274)$ \\
\hline Age (full range) & 61 (24-88) years & $60(21-86)$ years & $60(21-88)$ years \\
\hline \multicolumn{4}{|l|}{ Genotype } \\
\hline TTR mutation & 105 (63\%) & 71 (66\%) & $176(64 \%)$ \\
\hline V3OM & 101 (96\%) & 70 (99\%) & 171 (97\%) \\
\hline Other & $4(4 \%)$ & $1(1 \%)$ & $5(3 \%)$ \\
\hline $\begin{array}{l}\text { No TTR muta- } \\
\text { tion }\end{array}$ & $26(16 \%)$ & $17(16 \%)$ & $43(16 \%)$ \\
\hline Not tested & $36(21 \%)$ & $19(18 \%)$ & $55(20 \%)$ \\
\hline \multicolumn{4}{|c|}{ Symptom/finding ${ }^{\mathrm{a}}$} \\
\hline Polyneuropathy & 146 (87\%) & 100 (93\%) & $246(90 \%)$ \\
\hline $\begin{array}{l}\text { Cardiomyopa- } \\
\text { thy }\end{array}$ & $62(35 \%)$ & $17(16 \%)$ & 79 (29\%) \\
\hline Gl symptoms & $43(26 \%)$ & $41(38 \%)$ & $84(31 \%)$ \\
\hline $\begin{array}{l}\text { Eye complica- } \\
\text { tions }\end{array}$ & $21(13 \%)$ & $26(24 \%)$ & 47 (17\%) \\
\hline $\begin{array}{l}\text { Other symp- } \\
\text { toms }\end{array}$ & $1(0.5 \%)$ & $2(2 \%)$ & $3(1 \%)$ \\
\hline \multicolumn{4}{|l|}{ Echocardiogram } \\
\hline IVSd $\geq 12 \mathrm{~mm}$ & $68(72 \%)$ & $25(45 \%)$ & $93(62 \%)$ \\
\hline IVSd < 12 mm & $26(28 \%)$ & $31(55 \%)$ & 57 (38\%) \\
\hline \multicolumn{4}{|l|}{${ }^{99} \mathrm{~m}_{\mathrm{Tc}}-\mathrm{DPD}$ scint } \\
\hline Positive & $18(78 \%)$ & $6(46 \%)$ & $24(67 \%)$ \\
\hline Negative & $5(22 \%)$ & $7(54 \%)$ & $12(33 \%)$ \\
\hline $\begin{array}{l}\text { Amyloid in first } \\
\text { Bx }\end{array}$ & $70(42 \%)$ & 39 (36\%) & $109(40 \%)$ \\
\hline
\end{tabular}

${ }^{a}$ Each individual can present with one or more symptom/finding; Bx: biopsy; Gl: gastrointestinal; IVSd: interventricular septum diameter; scint: scintigraphy; TTR : transthyretin gene. Percentages are shown as column percentage within each heading/subheading

Table 2 Final diagnoses

\begin{tabular}{lccc}
\hline & Male (n=167) & Female (n=107) & All (n=274) \\
\hline No amyloidosis & $80(48 \%)$ & $62(58 \%)$ & $142(52 \%)$ \\
TTR mutation & $22(28 \%)$ & $31(50 \%)$ & $53(37 \%)$ \\
No TTR mutation & $58(72 \%)$ & $31(50 \%)$ & $89(63 \%)$ \\
Amyloidosis & $87(52 \%)$ & $45(42 \%)$ & $132(48 \%)$ \\
ATTRv amyloidosis & $82(94 \%)$ & $41(91 \%)$ & $123(93 \%)$ \\
Dx before 2006 & $10(12 \%)$ & $9(22 \%)$ & $19(16 \%)$ \\
ATTRV30M & $69(84 \%)$ & $31(76 \%)$ & $100(81 \%)$ \\
Other mutation ${ }^{\text {a }}$ & $3(4 \%)$ & $1(2 \%)$ & $4(3 \%)$ \\
ATTRwt amyloi- & $1(1 \%)$ & $0(0 \%)$ & $1(1 \%)$ \\
dosis & & $3(7 \%)$ & $7(5 \%)$ \\
AL amyloidosis & $4(5 \%)$ & $3(100 \%)$ & $6(86 \%)$ \\
Localized & $3(75 \%)$ & $0(0 \%)$ & $1(14 \%)$ \\
Systemic & $1(25 \%)$ & $1(2 \%)$ & $1(1 \%)$ \\
Gelsolin amyloi- & $0(0 \%)$ & & \\
dosis & & & \\
\hline
\end{tabular}

a H88R, A45S, V122I and A97S; AL, amyloid light chain; ATTRv, variant transthyretin amyloidosis; ATTRV30M, transthyretin amyloidosis caused by the TTR V30M mutation; ATTRwt, wild-type transthyretin amyloidosis; Dx, diagnosis; $T T R$, transthyretin gene. Percentages are shown as column percentage within each heading/subheading 
Data on typing was missing in five cases, and the amount of amyloid deposits was too small for typing in five cases.

\section{Specificity and sensitivity}

The overall test specificity was $99 \%$ and the overall sensitivity was $90 \%$ for patients with suspected systemic amyloidosis, which corresponded to positive and negative predictive values of 0.99 and 0.93 , respectively. Only one false positive biopsy was found in a previously healthy 44-year-old male patient with a painful peripheral neuropathy without signs of monoclonal gammopathy and no TTR mutation. The first histochemical analysis showed small amounts of amyloid in the abdominal fat, but further typing was not possible. Subsequent abdominal fat pad and upper GI tract biopsies showed no signs of amyloid, which led to the conclusion that the first fat pad biopsy had been contaminated.

Due to the limited number of patients with other diagnoses, subsequent analyses were focused on the remaining 104 patients with ATTRv amyloidosis. For these patients, the results were similar with a test specificity of $99 \%$ and a sensitivity of $91 \%$ (positive and negative predictive values of 0.99 and 0.94 , respectively). The initial abdominal fat pad biopsy was negative for amyloid in nine of the cases, predominantly males and late-onset patients (Table 3 ).

\section{Subgroup analyses within ATTRv amyloidosis patients}

No significant differences in test sensitivity were found between the sexes $(p=0.34)$, between early and lateonset cases $(p=0.66)$ or across amyloid fibril types $(p=0.58)$.

Among the 104 patients with ATTRv amyloidosis, the majority (94\%) had signs of peripheral polyneuropathy, whereas 55\% had signs of amyloid cardiomyopathy (based on echocardiograms and ${ }^{99 \mathrm{~m}} \mathrm{Tc}-\mathrm{DPD}$ scintigraphies that had been performed in $89 \%$ and $27 \%$ of the patients, respectively). Most patients had a mixed phenotype with both polyneuropathy and cardiomyopathy, and only six patients (five males) had a pure cardiac phenotype at the time of the first diagnostic biopsy. Test sensitivity in relation to the patients' sex and symptoms/findings is displayed in Fig. 2, and no significant differences were found between any of the subgroups. No negative biopsy results were found in the 15 female patients with ATTR cardiomyopathy, of whom all were late-onset cases, 13 had type A fibrils and 11 displayed a positive ${ }^{99 \mathrm{~m}} \mathrm{Tc}-\mathrm{DPD}$ scintigraphy.

\section{Discussion}

To the best of our knowledge, this is the first comprehensive evaluation of the accuracy of minimally invasive surgical fat pad biopsies for diagnosing systemic amyloidosis. The study was based on a relatively large patient material that was collected at a tertiary referral centre within the Swedish clustering area of ATTRV30M amyloidosis. Thus, the majority of patients with amyloidosis had been diagnosed with ATTRv amyloidosis, and the results should be interpreted accordingly. A clinical follow-up time of more than three years after the first diagnostic biopsy should have been sufficient to capture any false negative (or positive) biopsy results.

Overall, the diagnostic specificity and sensitivity were high (99\% and $91 \%$, respectively) and were not significantly different for any of the subgroups analysed. However, the sample sizes were too small to evaluate the diagnostic accuracy in patients with other types of amyloidosis than ATTRv amyloidosis. The number of patients was also low for some subgroups of ATTRv amyloidosis patients, especially regarding those with merely cardiac complications, which of course could have affected the outcome of the statistical analyses.

Table 3 Negative biopsy cases for nine patients among ATTRv amyloidosis patients diagnosed within three years from the initial biopsy

\begin{tabular}{|c|c|c|c|c|c|c|c|c|c|}
\hline & Sex & Age & Mut & Phenotype & Onset & Fibrils & 2nd fat Bx & Other Bx & Dx per \\
\hline 1 & $\mathrm{~F}$ & 74 & V30M & PN, eye & Late & NA & - & $\mathrm{CT}+$ & CT Bx \\
\hline 2 & M & 67 & V30M & PN & Late & NA & + & $\mathrm{Gl}+$ & Fat/Gl Bx \\
\hline 3 & M & 72 & V30M & PN, CM, Gl & Late & NA & + & ND & Fat Bx/DPD \\
\hline 4 & $\mathrm{~F}$ & 64 & V30M & PN, Gl, eye & Late & NA & ND & Skin+ & Skin Bx \\
\hline 5 & M & 66 & V122I & $\mathrm{CM}$ & Late & NA & ND & Heart+ & Heart Bx \\
\hline 6 & M & 62 & V30M & PN, CM & Late & Type B & + & ND & Fat Bx/DPD \\
\hline 7 & M & 58 & V30M & $P N, C M$ & Late & NA & - & ND & DPD \\
\hline 8 & M & 52 & V30M & PN, CM & Late & NA & + & ND & Fat Bx/DPD \\
\hline 9 & M & 46 & V30M & PN, Gl & Early & NA & - & Gl- & Clin. picture \\
\hline
\end{tabular}

Bx, biopsy; Clin, clinical; CM, cardiomyopathy; CT, carpal tunnel; DPD, ${ }^{99 m}$ Tc-DPD scintigraphy; Dx, diagnosis; F, female; Gl, gastrointestinal; M, male; Mut, mutation; NA, not available; ND, not done; PN, polyneuropathy; - , negative; + , positive 


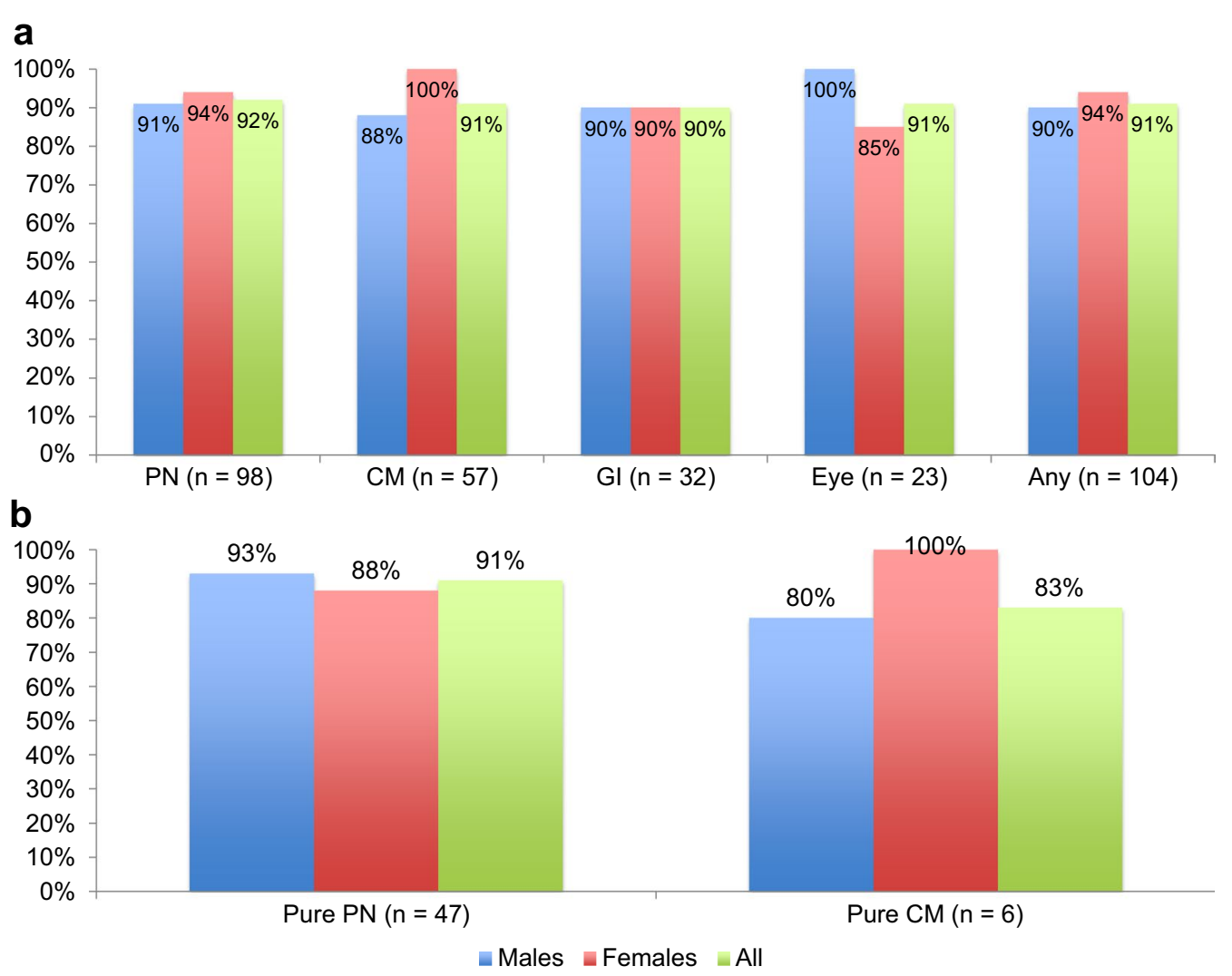

Fig. 2 Test sensitivity in ATTRv amyloidosis patients. Sensitivity of abdominal fat pad biopsies in different subgroups of patients with a final diagnosis of hereditary transthyretin (ATTRv) amyloidosis within three years from initial biopsy. a Test sensitivity in subgroups as per symptom/ findings and sex. Each patient can present with more than one symptom or complication, and most patients presented with a mixed phenotype. No significant differences were found between the subgroups. b Test sensitivity in patients with a pure neuropathic or pure cardiac phenotype. No significant differences were found between the groups. CM: cardiomyopathy; eye: eye complications; Gl: gastrointestinal symptoms; PN: polyneuropathy

The numerically lowest sensitivity was found in male patients with ATTR cardiomyopathy. This is probably related to the amyloid fibril type since type A fibrils is associated with this phenotype, and since the type A amyloid generally is more scarcely distributed outside the clinically affected organs [13]. Six (67\%) of the patients with false negative biopsies were late-onset males, and all except one had cardiomyopathy. Interestingly, no false negative biopsies were found in female patients with ATTR cardiomyopathy and type A fibrils. It is well recognised that men are more prone for cardiac ATTR deposition than women [23-27] and, thus, females may develop cardiomyopathy at later stages of the disease, which allows for more amyloid accumulation in non-cardiac tissues. Apart from this, we found no evidence of any differences in amyloid deposition in subcutaneous fat between the clinical phenotypes. However, it should be noted that patients with exclusively ocular or central nervous system (CNS) complications can present with negative peripheral tissue biopsies, although they suffer from oculoleptomeningeal ATTRv amyloidosis, since there is a local production of TTR in the eye and in the CNS.

In our material, only occasional patients with nonV30M mutations were included so no analyses of the impact of genotype on the diagnostic accuracy of abdominal fat pad biopsies were possible. Theoretically, the genotype could be of importance since it is linked to the disease phenotype and since most non-V30M mutations, as well as ATTRwt, seem to be associated with type A fibrils [28].

Only one false positive fat pad biopsy was found in the study; however, it is important to note that Congo red is not an amyloid-specific dye and that it can also stain foreign particles with amyloid properties as well as collagen and hyaline [29]. This can lead to false positive results, especially if the tissue sample is over stained and if the specimen is analysed at a laboratory with limited experience of amyloid diagnosis. An advantage of subcutaneous adipose tissue in this context is that it contains low levels of proteins compared to other tissues [15]. With the 
method used in the present study, the preparations are thicker compared to those generally used for microscopy, and Congo red stained deposits thereby appear more clearly and are more easily distinguishable from the background (Fig. 3). It is also our experience that ATTR amyloid, particularly of type A, shows a stronger stainability with Congo red if formalin fixation and paraffin embedment are avoided.

Nonetheless, contamination of the sample with for example textile fibres can be interpreted as amyloid deposits, which was probably the case in our patient; therefore, it is important to minimize the use of textile tissues and swabs during the biopsy procedure. Moreover, long-term subcutaneous injections of insulin due to diabetes mellitus can give rise to local insulin-derived amyloid deposits [30], which is of course important to be aware of when performing an abdominal fat pad biopsy for amyloid diagnosis on a patient with insulin treatment.

With the above in mind, it is evident that typing of the amyloid is central for verification of the precursor protein. We have developed in-house antibodies to use for typing with immunohistochemistry or Western blot, since commercial antibodies often fail to determine ATTR fragments $[15,20]$. However, proteomics and mass spectrometry have also been shown to be feasible alternatives [31]. From our experience, an abdominal fat pad biopsy using a skin punch is a safe and convenient way to obtain an adequate tissue sample that enables reliable detection of amyloid, as well as determination of the precursor protein and of the ATTR fibril type. In the current study, typing was possible in the majority of patients although the amount of amyloid was too small for determining the precursor protein and the fibril type in five cases. Typing with Western blot is usually not possible with biopsies from other organs, since the amount of tissue sent for histopathology is smaller and thereby also is the quantity of amyloid. However, mass spectrometry can be an alternative method for amyloid typing in these cases [32].

Currently at our centre, we primarily use surgically obtained abdominal fat pad biopsies for diagnosing systemic amyloidosis. We previously performed needle aspiration (using 16-20 Gauge needles) of abdominal fat after local anaesthesia, but have discarded this procedure since it was painful for the patient and difficult to obtain a sufficient amount of tissue for a reliable histopathological evaluation. In addition, blood contamination of the sample was a problem. However, good sensitivity has been

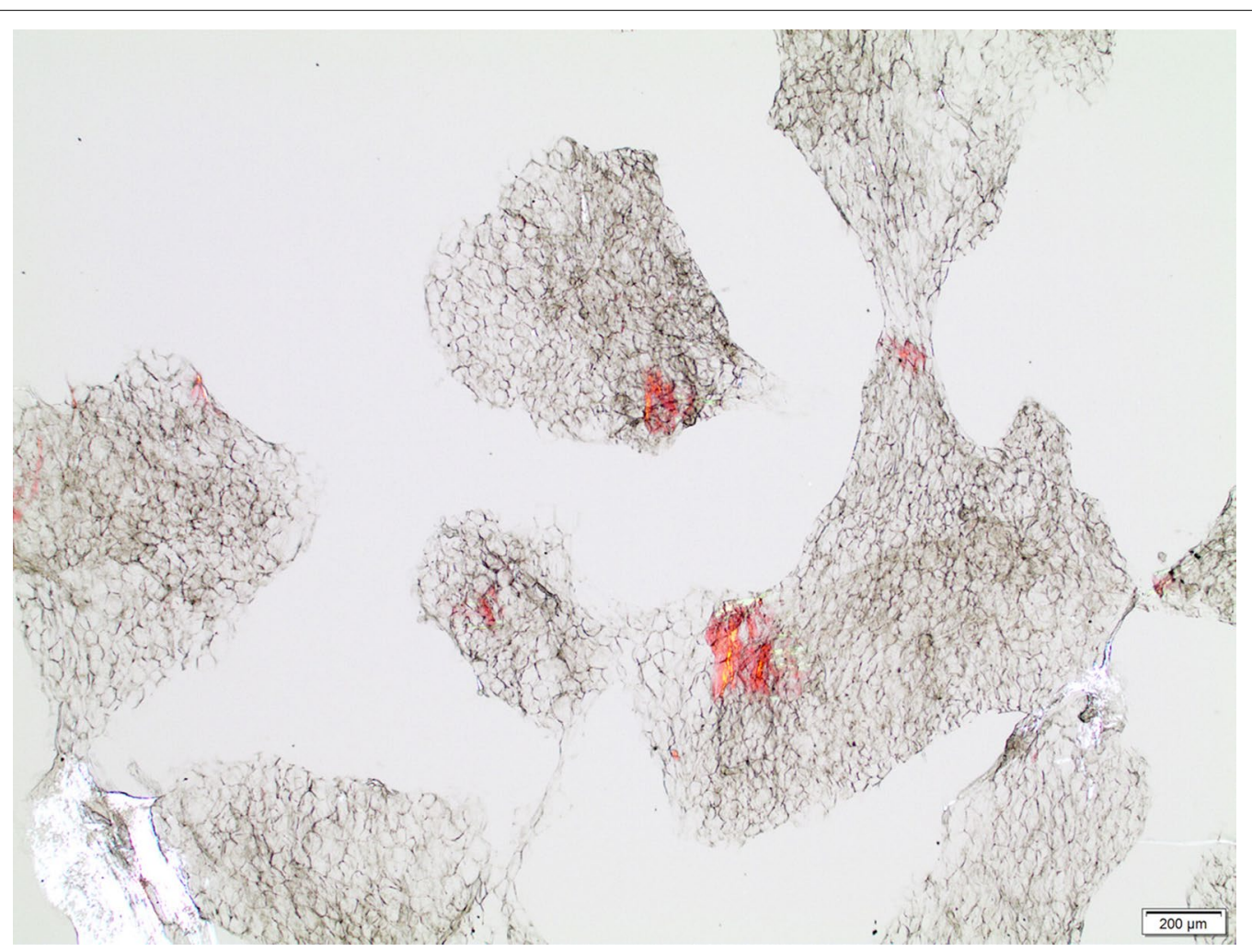

Fig. 3 Histopathology of adipose tissue with amyloid deposits. Adipose tissue biopsy from a patient with type A transthyretin amyloid fibrils. The spotty appearance of sharply demarcated deposits is characteristic. Such deposits are sometimes small and quite few, and a systematic review is necessary. Congo red staining, normal light. Bar $=200 \mu \mathrm{m}$ 
reported utilising this method if a sufficient amount of fat can be obtained [33]. The diagnostic sensitivity of fine needle aspirates was shown to be unreasonably low for patients with ATTR amyloidosis, especially for patients with ATTR cardiomyopathy [16, 17, 34]. Although skin punch biopsies are slightly more invasive than needle aspiration, we have not experienced a higher rate of complications with this technique. Therefore, we strongly suggest using skin punch biopsies, rather than fine needle aspiration, to obtain fatty tissue samples in patients with suspected ATTR amyloidosis.

Although the diagnostic sensitivity of the abdominal fat pad biopsies was high in our material, repeated fat pad biopsies and/or alternative biopsy sites were required to establish the diagnosis in some cases. This is probably difficult to avoid since the amyloid deposits can be scarce in early stages of the disease and also unevenly distributed in the tissue, especially in patients with type A ATTR fibrils $[16,35]$. It is also possible that ATTR amyloidosis has developed during the time between biopsies.

If amyloidosis is strongly suspected and the abdominal fat pad biopsy is negative for amyloid, we usually repeat the biopsy after six months or plan for a biopsy from another organ (e.g. GI tract or heart) depending on the clinical picture. The diagnostic accuracy for other commonly used biopsy sites, such as sural nerve, GI tract and labial salivary glands, is varying. Sural nerve biopsy has a reported test sensitivity of 73-87\% [36-38]; however, the procedure induces permanent nerve damage and is not a preferred site for diagnostic biopsies at our centre. GI tract biopsies was reported to have an average diagnostic sensitivity of $16 \%$ in a large retrospective study from Germany; however, the sensitivity increased to $99 \%$ if submucosal vessels were included in the biopsies [39]. Another retrospective analysis from the Mayo Clinic reported a diagnostic sensitivity of $81 \%$ for rectal biopsies in ATTR cardiac amyloidosis [16]. We occasionally perform gastroduodenal or rectal biopsies for diagnostic purposes at our centre. Labial salivary glands is the preferred biopsy site at many centres and has a reported sensitivity of $75 \%$ to $91 \%$, the latter achieved in a Portuguese population of generally early onset ATTRV30M patients $[40,41]$. We have no experience of this procedure but it is an acceptable diagnostic option, although it appears to be more uncomfortable for the patients than abdominal fat pad biopsies. Unlike adipose tissue biopsies, all of the abovementioned techniques require fixation, paraffin-embedding and sectioning of the tissue. By avoiding these steps, type A ATTR is more strongly stained with Congo red, and small amyloid deposits are more easily identified in the thicker (up to $100 \mu \mathrm{m}$ ) adipose tissue fragments obtained with our method. In the case of suspect ATTR cardiomyopathy, bone scintigraphy can be a good alternative to a tissue biopsy as long as a monoclonal gammopathy has been ruled out [18]. However, it should be noted that the sensitivity for bone scintigraphy is low for patients with type B ATTR fibrils [42].

In Sweden, we normally require demonstration of amyloid deposits in a tissue biopsy to establish the diagnosis of ATTR amyloidosis, even in patients with a proven $T T R$ mutation and typical symptoms of amyloidosis. This is due to the low penetrance of ATTRv amyloidosis in the Swedish population [43], and due to our rather old patient population with other potential causes of both polyneuropathy and heart disease. Therefore, tissue biopsies are liberally performed, especially in regard to $T T R$ mutation carriers. Since the ATTR fibril type is associated with the disease phenotype and with the survival after liver transplantation $[13,14]$, we also find it essential to try to establish the amyloid fibril type. It remains to be elucidated whether the amyloid fibril type also has any implications on the response to other therapies, such as TTR stabilisers and TTR gene silencing.

\section{Conclusions}

Abdominal fat pad biopsies performed with an $8 \mathrm{~mm}$ skin punch exhibit good diagnostic accuracy in patients with suspect ATTRv amyloidosis when histopathology is performed at a specialized centre. Further, test sensitivity was not significantly lower for patients with cardiomyopathy or for other subgroups of patients. We therefore conclude that abdominal fat pad biopsies is a safe and reliable diagnostic tool in ATTRv amyloidosis that enables typing of the precursor protein and of the amyloid fibril type.

\section{Supplementary information}

Supplementary information accompanies this paper at https://doi. org/10.1186/s13023-020-01565-8.

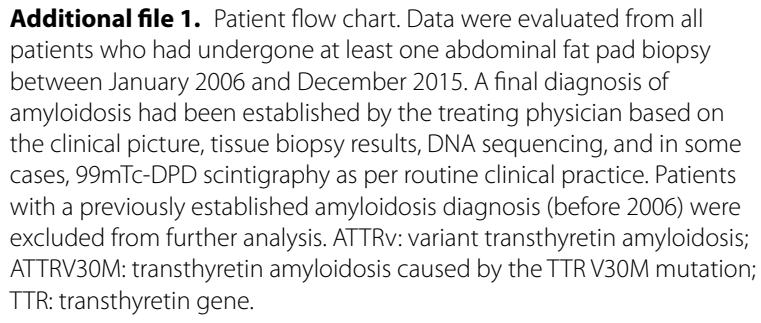

Additional file 1. Patient flow chart. Data were evaluated from all patients who had undergone at least one abdominal fat pad biopsy between January 2006 and December 2015. A final diagnosis of amyloidosis had been established by the treating physician based on the clinical picture, tissue biopsy results, DNA sequencing, and in some cases, 99mTc-DPD scintigraphy as per routine clinical practice. Patients with a previously established amyloidosis diagnosis (before 2006) were excluded from further analysis. ATTRv: variant transthyretin amyloidosis; ATTRV30M: transthyretin amyloidosis caused by the TTR V30M mutation; TTR: transthyretin gene.

\section{Acknowledgements}

We sincerely thank and acknowledge all the patients who contributed with data for this study.

\section{Authors' contributions}

HPR and NSG reviewed the patients' medical records, analysed and interpreted the data and were major contributors in writing the manuscript. PW and OBS contributed to the data collection, participated in the planning of the study and revised the manuscript. IA, El and BP contributed to the data collection 
and revised the manuscript. JW contributed to the data collection, participated in the planning of the study, and contributed with analysis and interpretation of data, as well as with writing of the manuscript. All authors read and approved the final manuscript.

\section{Funding}

Open Access funding provided by University Of Umea. The study was funded by research grants from the Region of Västerbotten, the Swedish Heart-Lung Foundation grant no 20160787, the Swedish Research Council grant no 201901338, the Knut and Alice Wallenberg Foundation, Selander's Foundation, the Patients' Organizations FAMY, FAMY Norrbotten and AMYL.

\section{Availability of data and materials}

The datasets used and/or analysed during the current study are available from the corresponding author on reasonable request.

\section{Ethics approval and consent to participate}

Ethical approval was obtained from the ethical review board of Umea University, reference number 2017-447-31. No specific consent to participate in the study was considered necessary.

\section{Consent for publication}

Not applicable.

\section{Competing interests}

The authors declare that they have no competing interests.

\section{Author details}

${ }^{1}$ Department of Public Health and Clinical Medicine, Umeå University, 901 87 Umeå, Sweden. ${ }^{2}$ Department of Immunology, Genetics and Pathology, Uppsala University, Uppsala, Sweden. ${ }^{3}$ Wallenberg Centre for Molecular Medicine, Umeå University, Umeå, Sweden.

Received: 17 June 2020 Accepted: 25 September 2020

Published online: 08 October 2020

\section{References}

1. Pinney JH, Hawkins PN. Amyloidosis. Ann Clin Biochem. 2012;49:229-41.

2. Kyle RA, Bayrd ED. Amyloidosis: review of 236 cases. Medicine (Baltimore). 1975:54:271-99.

3. Westermark P. Diagnosing amyloidosis. Scand J Rheumatol. 1995:24:327-9.

4. Röcken C, Schwotzer EB, Linke RP, Saeger W. The classification of amyloid deposits in clinicopathological practice. Histopathology. 1996;29:325-35.

5. Brambilla F, Lavatelli F, Di Silvestre D, Valentini V, Rossi R, Palladini G, et al. Reliable typing of systemic amyloidoses through proteomic analysis of subcutaneous adipose tissue. Blood. 2012;119:1844-7.

6. Quintas A, Vaz DC, Cardoso I, Saraiva MJ, Brito RM. Tetramer dissociation and monomer partial unfolding precedes protofibril formation in amyloidogenic transthyretin variants. J Biol Chem. 2001;276:27207-13.

7. Wiseman RL, Powers ET, Kelly JW. Partitioning conformational intermediates between competing refolding and aggregation pathways: insights into transthyretin amyloid disease. Biochemistry. 2005;44:16612-23.

8. Tanskanen M, Peuralinna T, Polvikoski T, Notkola I-L, Sulkava R, Hardy J, et al. Senile systemic amyloidosis affects $25 \%$ of the very aged and associates with genetic variation in alpha2-macroglobulin and tau: a population-based autopsy study. Ann Med. 2008;40:232-9.

9. Ueda M, Horibata Y, Shono M, Misumi Y, Oshima T, Su Y, et al. Clinicopathological features of senile systemic amyloidosis: an ante- and postmortem study. Mod Pathol. 2011;24:1533-44.

10. Planté-Bordeneuve V, Said G. Familial amyloid polyneuropathy. Lancet Neurol. 2011;10:1086-97.

11. Parman Y, Adams D, Obici L, Galán L, Guergueltcheva V, Suhr OB, et al. Sixty years of transthyretin familial amyloid polyneuropathy (TTR-FAP) in Europe: where are we now? A European network approach to defining the epidemiology and management patterns for TTR-FAP. Curr Opin Neurol. 2016;29(Suppl 1):S3-13.
12. Rowczenio DM, Noor I, Gillmore JD, Lachmann HJ, Whelan C, Hawkins PN, et al. Online registry for mutations in hereditary amyloidosis including nomenclature recommendations. Hum Mutat. 2014;35:E2403-2412.

13. Suhr $\mathrm{OB}$, Lundgren $\mathrm{E}$, Westermark P. One mutation, two distinct disease variants: unravelling the impact of transthyretin amyloid fibril composition. J Intern Med. 2017;281:337-47.

14. Ericzon B-G, Wilczek HE, Larsson M, Wijayatunga P, Stangou A, Pena $J R$, et al. Liver transplantation for hereditary transthyretin amyloidosis: after 20 years still the best therapeutic alternative? Transplantation. 2015;99:1847-54.

15. Westermark P, Davey E, Lindbom K, Enqvist S. Subcutaneous fat tissue for diagnosis and studies of systemic amyloidosis. Acta Histochem. 2006;108:209-13.

16. Fine NM, Arruda-Olson AM, Dispenzieri A, Zeldenrust SR, Gertz MA, Kyle RA, et al. Yield of noncardiac biopsy for the diagnosis of transthyretin cardiac amyloidosis. Am J Cardiol. 2014;113:1723-7.

17. Quarta CC, Gonzalez-Lopez E, Gilbertson JA, Botcher N, Rowczenio $D$, Petrie A, et al. Diagnostic sensitivity of abdominal fat aspiration in cardiac amyloidosis. Eur Heart J. 2017;38:1905-8.

18. Gillmore JD, Maurer MS, Falk RH, Merlini G, Damy T, Dispenzieri A, et al. Nonbiopsy diagnosis of cardiac transthyretin amyloidosis. Circulation. 2016;133:2404-12.

19. Westermark P. Subcutaneous adipose tissue biopsy for amyloid protein studies. Methods Mol Biol. 2012;849:363-71.

20. Westermark GT, Ihse E, Westermark P. Development of mouse monoclonal antibodies against human amyloid fibril proteins for diagnostic and research purposes. Methods Mol Biol. 2018;1779:401-14.

21. Perugini E, Guidalotti PL, Salvi F, Cooke RMT, Pettinato C, Riva L, et al. Noninvasive etiologic diagnosis of cardiac amyloidosis using 99mTc3,3-diphosphono-1,2-propanodicarboxylic acid scintigraphy. J Am Coll Cardiol. 2005:46:1076-84.

22. Wixner J, Suhr OB, Anan I. Management of gastrointestinal complications in hereditary transthyretin amyloidosis: a single-center experience over 40 years. Expert Rev Gastroenterol Hepatol. 2018;12:73-81.

23. Hörnsten R, Pennlert J, Wiklund U, Lindqvist P, Jensen SM, Suhr OB. Heart complications in familial transthyretin amyloidosis: impact of age and gender. Amyloid. 2010;17:63-8.

24. Tasaki M, Ueda M, Obayashi K, Koike H, Kitagawa K, Ogi Y, et al. Effect of age and sex differences on wild-type transthyretin amyloid formation in familial amyloidotic polyneuropathy: a proteomic approach. Int J Cardiol. 2013;170:69-74.

25. Rapezzi C, Quarta CC, Obici L, Perfetto F, Longhi S, Salvi F, et al. Disease profile and differential diagnosis of hereditary transthyretin-related amyloidosis with exclusively cardiac phenotype: an Italian perspective. Eur Heart J. 2013;34:520-8.

26. Arvidsson S, Pilebro B, Westermark P, Lindqvist P, Suhr OB. Amyloid cardiomyopathy in hereditary transthyretin $\mathrm{V} 30 \mathrm{M}$ amyloidosis_impact of sex and amyloid fibril composition. PLoS ONE. 2015;10:e0143456.

27. Mohamed-Salem L, Santos-Mateo JJ, Sanchez-Serna J, HernándezVicente Á, Reyes-Marle R, Castellón Sánchez MI, et al. Prevalence of wild type ATTR assessed as myocardial uptake in bone scan in the elderly population. Int J Cardiol. 2018;270:192-6.

28. Ihse E, Rapezzi C, Merlini G, Benson MD, Ando Y, Suhr OB, et al. Amyloid fibrils containing fragmented ATTR may be the standard fibril composition in ATTR amyloidosis. Amyloid. 2013;20:142-50.

29. Fernandez-Flores A. A review of amyloid staining: methods and artifacts. Biotech Histochem. 2011:86:293-301.

30. Katzman BD, Traum P, Medline PB. New histologic finding of amyloid insulin bodies at an insulin injection site in a patient with diabetes. Am J Dermatopathol. 2018:40:527-30.

31. Picken MM. Proteomics and mass spectrometry in the diagnosis of renal amyloidosis. Clin Kidney J. 2015;8:665-72.

32. Klein CJ, Vrana JA, Theis JD, Dyck PJ, Dyck PJB, Spinner RJ, et al. Mass spectrometric-based proteomic analysis of amyloid neuropathy type in nerve tissue. Arch Neurol. 2011;68:195-9.

33. van Gameren II, Hazenberg BPC, Bijzet J, van Rijswijk MH. Diagnostic accuracy of subcutaneous abdominal fat tissue aspiration for detecting systemic amyloidosis and its utility in clinical practice. Arthritis Rheum. 2006;54:2015-21. 
34. Fernández de Larrea C, Verga L, Morbini P, Klersy C, Lavatelli F, Foli A, et al. A practical approach to the diagnosis of systemic amyloidoses. Blood. 2015;125:2239-44.

35. Ikeda S, Sekijima Y, Tojo K, Koyama J. Diagnostic value of abdominal wall fat pad biopsy in senile systemic amyloidosis. Amyloid. 2011;18:211-5.

36. Planté-Bordeneuve V, Ferreira A, Lalu T, Zaros C, Lacroix C, Adams D, et al. Diagnostic pitfalls in sporadic transthyretin familial amyloid polyneuropathy (TTR-FAP). Neurology. 2007;69:693-8.

37. Cappellari M, Cavallaro T, Ferrarini M, Cabrini I, Taioli F, Ferrari S, et al. Variable presentations of TTR-related familial amyloid polyneuropathy in seventeen patients. J Peripher Nerv Syst: JPNS. 2011;16:119-29.

38. Koike H, Hashimoto R, Tomita M, Kawagashira Y, lijima M, Tanaka F, et al. Diagnosis of sporadic transthyretin Val30Met familial amyloid polyneuropathy: a practical analysis. Amyloid. 2011;18:53-62.

39. Freudenthaler S, Hegenbart U, Schönland S, Behrens H-M, Krüger S, Röcken C. Amyloid in biopsies of the gastrointestinal tract-a retrospective observational study on 542 patients. Virchows Arch Int J Pathol. 2016;468:569-77.
40. Do Amaral B, Coelho T, Sousa A, Guimarães A. Usefulness of labial salivary gland biopsy in familial amyloid polyneuropathy Portuguese type. Amyloid. 2009;16:232-8.

41. de Paula EF, de Mello BL, de Carvalho DLC, Della-Guardia B, de Almeida $M D$, Marins LV, et al. Minor salivary gland biopsy for the diagnosis of familial amyloid polyneuropathy. Neurol Sci. 2017;38:311-8.

42. Pilebro B, Suhr OB, Näslund U, Westermark $P$, Lindqvist $P$, Sundström T. (99m)Tc-DPD uptake reflects amyloid fibril composition in hereditary transthyretin amyloidosis. Upsala J Med Sci. 2016;121:17-24.

43. Hellman U, Alarcon F, Lundgren H-E, Suhr OB, Bonaiti-Pellié C, Planté-Bordeneuve $\mathrm{V}$. Heterogeneity of penetrance in familial amyloid polyneuropathy, ATTR Val30Met, in the Swedish population. Amyloid. 2008;15:181-6.

\section{Publisher's Note}

Springer Nature remains neutral with regard to jurisdictional claims in published maps and institutional affiliations.
Ready to submit your research? Choose BMC and benefit from:

- fast, convenient online submission

- thorough peer review by experienced researchers in your field

- rapid publication on acceptance

- support for research data, including large and complex data types

- gold Open Access which fosters wider collaboration and increased citations

- maximum visibility for your research: over $100 \mathrm{M}$ website views per year

At BMC, research is always in progress.

Learn more biomedcentral.com/submissions 